\title{
An interface of Chlamydia testing by community family planning clinics and referral to hospital genitourinary medicine clinics
}

Chris Wilkinson, MFFP, Consultant in Women's Sexual Health; Honorary Senior Lecturer in Gynaecology and Genitourinary Medicine, Kings College Hospital, London, UK

Helen Massil, MRCOG, MFFP, Consultant in Family Planning and Reproductive Healthcare; Jacqueline Evans, MRCGP, Staff Grade, Department of Family Planning, Community Health South London NHS Trust,

St. Giles Hospital, London, UK

Correspondence: Dr J Evans, Department of Family Planning and Reproductive Healthcare Community Health South London NHS Trust, St. Giles Hospital, St. Giles Rd., London, SE5 7RN, UK.

(Accepted March $\left.28^{\text {th }}, 2000\right)$

\begin{abstract}
Summary
Objectives. To assess compliance with the protocolfor the management of women with Chlamydia trachomatis diagnosed in community family planning (FP) clinics; to assess the rate of attendance at genitourinary medicine (GUM) clinics by these women; to assess the rate of adequate treatment and to assess the level of communication between GUM clinics and FP clinics.

Method. Retrospective review of FP clinic records and case notes to identify all women with positive or equivocal Chlamydia results during a 6 month period, and a retrospective review of records from five local GUM clinics. Results. One hundred and twelve women were identified from FP clinic records with positive or equivocal Chlamydia results. Eighty-nine (79.5\%) were referred to a GUM clinic. Twelve out of 14 women not referred had equivocal results. The median delay from the test being taken to the results being seen by a doctor was 9 days, and to the woman being referred was 10 days. Fifty-eight $(51.7 \%, n=112)$ women definitely attended a local GUM clinic. The FP clinics provided a letter of referral in 76 $(85.4 \%, n=89)$ women and the GUM clinics provided $a$ letter of reply in $21(48.8 \%, n=43)$ women who attended with a referral letter. Three months after testing, only 54 $(48.2 \%)$ of the 112 women with positive or equivocal Chlamydia tests were known by the referring FP clinic to have been treated.

Conclusions. The majority of women with positive or equivocal Chlamydia results were referred to a GUM clinic according to the protocol. Attendance at GUM clinics was disappointing, as only $51.7 \%$ of the 112 women with positive or equivocal results had documented evidence of having attended. This raises the question not whether community clinics should be testing, but whether they should be initiating treatment and partner notification. Collaborative work between GUM clinics and community clinics around partner notification is needed, as well as funding for training and additional pharmacy costs. Further collaborative work between GUM and FP and reproductive healthcare $(\mathrm{RHC})$ to evaluate the role of community clinics in the diagnosis and management of chlamydial infection and other sexually transmitted infections (STIs) is needed.
\end{abstract}

\section{Key words}

adequate treatment, Chlamydia trachomatis, community clinics, compliance with protocol, family planning clinics, interface between FP and GUM, referral to and attendance at GUM clinics, sexual health

\section{Key message points}

- The majority of women with positive or equivocal Chlamydia results in FPCs were referred to a GUM clinic according to protocol.

- Attendance at GUM clinics was disappointing. Only $51.7 \%$ of women with positive or equivocal Chlamydia results had documented evidence of attendance.

- Treatment of Chlamydia and partner notification in the community therefore need to be addressed.

- To ensure patients are managed to an acceptable standard collaborative work between GUM and FP around partner notification is needed.

- Further collaborative work is needed between GUM and FP to evaluate the role for community clinics in the diagnosis and management of chlamydial infection and other STIs.

\section{Introduction}

Genital Chlamydia infection is the most common treatable bacterial STI in England and Wales. It is often asymptomatic (in at least $70 \%$ of women and $50 \%$ of men), and consequences may be serious. Complications are more common in women than in men. It is one of the main causes of pelvic inflammatory disease (PID) in the developed world. PID can result in ectopic pregnancy, infertility, and chronic pelvic pain, all of which cause significant morbidity and are expensive to manage. ${ }^{1-4}$

Until recently most testing, treatment and partner notification for Chlamydia has occurred in hospital based GUM clinics. With the introduction of widely available diagnostic tests, more testing is now occurring in the community. Prevalence studies in the UK among women in different health care settings have shown there is a substantial level of asymptomatic infection among those generally perceived to be at low risk of a STI. ${ }^{2,4}$ Estimates of prevalence of genital Chlamydia infection in women attending UK FP clinics range from 3-7\%,.$^{3,4} \mathrm{FP}$ clinics are an appropriate place for testing as they serve a young, sexually active patient population. It is currently recommended that patients diagnosed as having Chlamydia in the community are referred to GUM clinics for treatment, full STI screening and contact tracing. ${ }^{4}$

South East London has high rates of both STI and termination of pregnancy (TOP), making effective treatment of Chlamydia in this area particularly vital. This audit was undertaken to assess current practice in the management of women with Chlamydia diagnosed in South East London community FP clinics. This is the first part of the audit cycle and a re-audit is currently underway. The 
Table 1 Time interval between testing and result seen, woman referred and attendance at GUM clinic

\begin{tabular}{lccc}
\hline & $\begin{array}{c}\text { Result seen } \\
\text { by doctor }\end{array}$ & $\begin{array}{c}\text { Referral by } \\
\text { doctor }\end{array}$ & $\begin{array}{c}\text { Attendance at } \\
\text { GUM clinic }\end{array}$ \\
\hline $\begin{array}{l}\text { Median time from } \\
\text { test in days (range) }\end{array}$ & $9(5-42)$ & $10(5-111)$ & $20(2-118)$ \\
$\begin{array}{l}\text { Median time from } \\
\text { referral in days (range) }\end{array}$ & - & - & $7.5(1-86)$ \\
$\begin{array}{l}\text { Total number } \\
\text { completed }(\mathrm{n}=12)\end{array}$ & $112(100 \%)$ & $89(79.5 \%)$ & $58(51.7 \%)$ \\
\hline
\end{tabular}

Department of Family Planning, Community South London NHS Trust (formerly known as Optimum NHS Trust), provides Chlamydia testing from 22 clinic sites using micro-track enzyme immunoassay (EIA). Chlamydia testing is offered to patients requesting TOP, and selectively prior to IUD fitting, as well as to symptomatic women. The existing protocol for the management of women with chlamydial infection advises referral of women with either equivocal or positive Chlamydia results to one of the five local GUM clinics. This protocol was developed at a departmental doctors' meeting and was based on generally accepted best practice at the time. The GUM clinics used a number of different Chlamydia testing methods at the time of this audit. All women referred to a GUM clinic should be given a standard clinic referral letter and GUM referral should be recorded in the woman's notes. The adherence to, and the efficacy of, this protocol have not previously been assessed.

\section{Aims}

The aims of the audit were to:

- assess FP clinic compliance with the protocol for the management of women with Chlamydia diagnosed in the community

- assess the rate of attendance at GUM clinics by these women

- assess the rate of adequate treatment

- assess the level of communication between GUM clinics and FP clinics.

\section{Method}

Cases (women with positive or equivocal Chlamydia EIA test results) were identified by a review of FP clinic records from 22 clinic sites between July and December 1995 by FP clinic staff. Data were collected by FP clinic staff or by the researcher who worked within one of the local GUM departments. GUM data were not fed back to the originating FP clinic during the study. Data collected on each case from the FP clinic notes included:

- result of the test (positive or equivocal)

- time between test being taken and result being seen by doctor

- time between test being taken and woman being referred to GUM clinics

- how women were informed of their result and referred to GUM clinics

- number of letters of reply from GUM clinics

- number of women known to have been treated 3 months after having test.

Cases were searched for and matched in the five local GUM clinics by date of birth, name and postcode. Data collected for each case included:
- date of first attendance

- recorded source of attendance

- whether anti-chlamydial treatment was given

- number of letters of reply sent to referring FP clinic.

\section{Results}

Total number of positive or equivocal Chlamydia results One hundred and twelve women had a positive or equivocal Chlamydia test (90 were positive and 22 were equivocal) during the 6 months of the audit.

The median age of these women was 21 years (age range 13-44 years).

Results seen by doctor

A doctor saw all Chlamydia results. The median time interval between testing and the result being seen by a doctor was 9 days (range 5-42 days). Sixty-four results $(57.1 \%)$ had been seen 2 weeks after the test was taken.

\section{Referral by doctor}

Out of 112 women with positive or equivocal Chlamydia tests, it was documented that 89 were referred $(79.5 \%), 14$ $(12.5 \%)$ were not referred and in nine $(8 \%)$ referral status was unknown.

It took a median of 10 days from testing to referral (range 5-111 days). Fifty-seven (50.9\%) of women had been referred 2 weeks after the test was taken.

Of the 14 women known not to have been referred, two had positive test results and 12 had equivocal test results. Reasons for not referring included equivocal tests being repeated in FP clinics, recent past history of treatment with antibiotics (by GP/gynaecology department/TOP clinic) and patient being unwilling to accept referral. In three cases reasons for non-referral were not documented.

Attendance at GUM clinic

Fifty-eight women definitely attended a GUM clinic $(51.7 \%, \mathrm{n}=112): 55$ women were identified from GUM clinic records and another three women had letters from GUM clinics in their FP clinic records. Attendance was suspected, but not confirmed, in another six women. Attendance was suspected when the woman had verbally reported attendance at a GUM clinic to the FP clinic, but no documentary evidence could be found.

The median time from the test to attendance at a GUM clinic was 20 days (range 2-118 days) and from referral to attendance at a GUM clinic was 7.5 days (range 1-86 days).

Thirty women $(33.7 \%$ of those referred) attended a GUM clinic within 3 weeks of the test being taken, and 35 women (39.3\% of those referred) attended a GUM clinic within 3 weeks of being referred by a doctor.

\section{Communication between FP clinics and GUM clinics}

A letter of referral was provided by the FP clinic in 76 women $(85.4 \%, \mathrm{n}=89$ women referred); 17 were handed to the women and 59 were posted.

Forty-three women who attended a GUM clinic had a letter of referral $(78.2 \%, \mathrm{n}=55$ women attending GUM clinic). Of the 43 women who attended a GUM clinic with a letter of referral, a letter was returned in only $21(48.8 \%$, $\mathrm{n}=43$ ).

\section{Treatment}

Three months after testing, only $54(48.2 \%)$ of the 112 women with positive or equivocal Chlamydia tests were known by the referring clinic to have been treated. This represents $60.6 \%$ of those referred $(n=89)$. 
Table 2 Number of women with positive or equivocal Chlamydia results tested

\begin{tabular}{|c|c|c|c|}
\hline Data source & Number treated & $\begin{array}{c}\text { Number treated as } \\
\text { a } \% \text { of total number } \\
\text { of definite referrals }(n=89)\end{array}$ & $\begin{array}{c}\text { Number treated as } \\
\text { a } \% \text { of total number } \\
\text { of positive and equivocal results }(n=112)\end{array}$ \\
\hline $\begin{array}{l}\text { FP clinic and GUM } \\
\text { data (documented } \\
\text { evidence of } \\
\text { attendance at GUM) }\end{array}$ & 54 & 60.6 & 48.2 \\
\hline $\begin{array}{l}\text { GUM data } \\
\text { (documented evidence } \\
\text { of attendance at GUM) }\end{array}$ & 51 & 57.3 & 45.5 \\
\hline $\begin{array}{l}\text { FP clinic data } \\
\text { (documented and verbal } \\
\text { evidence from women } \\
\text { of attendance at GUM) }\end{array}$ & 43 & 48.3 & 38.4 \\
\hline
\end{tabular}

\section{Discussion}

The majority of women with positive or equivocal Chlamydia results were referred to a GUM clinic according to the protocol. However, there was poor adherence to the protocol for referring women with equivocal results, demonstrating a need for further training of FP clinic staff in the management of equivocal results. Management of equivocal results is debatable, but the existing policy within the FP department was to refer such patients to a GUM department. This policy was devised after discussion with the local GUM services.

The median time taken for the results to be seen by a doctor and the women to be referred was acceptable ( 9 and 10 days, respectively), but in a small number of cases the delay was considerable. In at least one case this was due to the woman not returning for the results and giving no means of contact. Clinic sites where there is only one clinic session a week may also contribute to the length of time taken to see the results and refer. Those clinics with a session on a Monday are also prone to delay due to Bank Holidays. The mechanism by which an individual woman is to be informed of her result needs to be discussed and clearly documented in the notes.

Attendance at GUM clinics was disappointing, as only $52.7 \%$ of the 112 women with positive or equivocal Chlamydia results had documentary evidence of having attended. This figure may be an underestimate for the following reasons. Women may have attended using a false name, or they could have attended a clinic outside the area. It may also be that those not attending did not realise the importance of the infection, or that they did not want to attend a GUM clinic. It is also of note that three women were not treated in the GUM clinic as the client did not attend with a letter of referral and the GUM clinics' Chlamydia test was either negative (two subjects) or equivocal (one subject). Overall, the rates of women known to have been treated were low; only $48 \%$ of the original 112 women had documented evidence of appropriate treatment being given.

Traditionally, community FP clinics have not taken on a role in the diagnosis and treatment of sexually transmitted infections. EIA and DNA amplification tests for Chlamydia that can be taken geographically away from the laboratory without a loss in sensitivity have facilitated testing in community settings, including general practice. As such testing in community clinics is becoming more routine, this audit highlights the importance of ensuring that the mechanisms to manage patients with positive results are in place. It appeared that a number of the patients in this audit did not actually benefit from having the infection diagnosed, as they may not have had correct treatment or partner notification.

This also raises the question not whether community clinics should be testing, but whether they should also be initiating treatment and partner notification. For this to take place the ability to manage patients to an acceptable standard needs to be in place. To improve the situation collaborative work between GUM clinics and community clinics around partner notification is needed, as well as funding for training and additional pharmacy costs.

The CMO's Expert Advisory Group on Chlamydia trachomatis has highlighted the importance of community services in the diagnosis and management of Chlamydial infection. In light of this, and the advent of less invasive methods of diagnosis, it is important for us to ensure that existing policies and practices are effective. This audit therefore focuses on these aspects and, in particular, on the practical interface between FP and GUM and rather than trying to measure the prevalence of infection and the value of screening.

\section{Recommendations}

- Clinic staff should receive further training in the management of equivocal Chlamydia results. This should result in all women with equivocal Chlamydia results being referred to a GUM clinic.

- Clinic administrators should ensure that all Chlamydia results are seen by a doctor within 2 weeks of the test being taken. Clinics should run regular audits to assess whether this is happening and to take action to rectify this if it is not.

- The woman and clinic staff should agree how the woman will be informed of her results and this should be documented in the notes. If the woman is unable to give a contact address, the clinic staff should make sure that the woman understands that it is her responsibility to collect the result and the possible consequences if she fails to do this. A woman with a positive or equivocal result should receive a referral letter to a GUM clinic, a list of local GUM clinics and a patient information leaflet on Chlamydia. This should also be documented in the notes.

- GUM clinic staff should be encouraged to send letters confirming attendance and treatment if the woman has attended with a referral letter, to further improve communication between the referring clinic/ doctor and the GUM department. 
Acknowledgements

This work was co-ordinated by the Department of Genitourinary Medicine, St. Thomas Hospital, London, SE1, UK.

Statements on funding and competing interests

Funding. This project was supported by a grant from CAASC for interface audits

Competing interests. None declared.
Cates W, Wasserheit JN. Genital Chlamydia infections: Epidemiology and reproductive sequelae. Am J Obstet Gynecol 1991, 164: 1771-781

of genital Chlamydia trachomatis in England and Wales. Genitourinary Medicine 1997; 73: 122-126.

C.

Chief Medical Officer. Main report of the Chief Medical Officer's Expert Advisory Group o Chlamydia trachomatis. London: Department of Health, 1998.

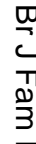

